\title{
FIBER FED SPECTROGRAPH FOR LINE VARIABILITY STUDIES
}

\author{
P. Felenbok and J. Guérin \\ Observatoire de Paris, Section de Meudon
}

Introduction : In our studies of activity in pre-main sequence Herbig $\mathrm{Ae} / \mathrm{Be}$ stars we are mainly interested in searching rotational modulation of line profile. If the period of star rotation is of the order of one or two days, the data collected from a single site is insufficient. This led us to start correlated observations from two or three sites spread as much as possible in longitude . Our first bi-site observations started in 1982 on AB Aur from two observatories located 11 hours apart.: CFHT in Hawaï and OHP in France. To achieve a high flexibility and to gain access to telescopes without attached spectrographs. We built an instrument that is mobile and specially designed for line profile studies.

The spectrograph (ISIS): The optical design is of the Czerny-Turner perpendicular type which is a pure Littrow mount giving a high luminosity in the blaze angle. Fig. 1 shows the spectrograph and his link to the telescope.This instrument is coupled to any kind of telescope by an optical fiber. We use step index silica-silica fibers :vith $133 \mu \mathrm{m}$ or $200 \mu \mathrm{m}$ core. In order to take into account the fiber aperture degradatios the fiber is fed with a telescope beam at $\mathrm{f} / 5$ and the spectrograph collimator is a parabola on axis at $\mathrm{f} / 3.7$. Two gratings are mounted in a flipping barrel, one for a resolution $R=$ $3.510^{4}$ and the other one for $R=10^{4}$. The optical quality of the instrument, with the slit parallel to the grating grooves was checked on photographic plates in a $36 \mathrm{~mm}$ field, far bigger than the CCD's surface. Fig. 2 shows the test spectrum taken with a thorium electrodeless discharge lamp.As it is seen on fig. 2, our optical design is generating tilted spectral lines with respect to the direction of dispersion. This is handled quite well with standard reduction software and cannot be avoided if the uttermost resolution is required. 

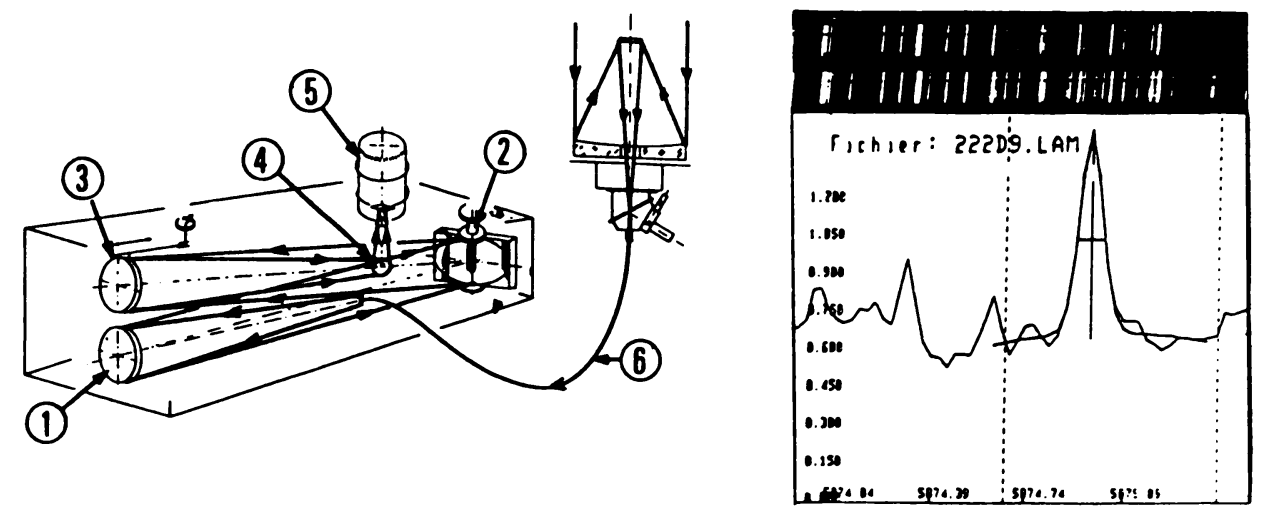

Eig. 1: The optical design of the ISIS spectrograph

1) Parabolic collimator $\varnothing 160 \mathrm{~mm} \quad f=600 \mathrm{~mm}$

2) Gratings -a $600 \mathrm{gr} / \mathrm{mm} \mathrm{B} \mathrm{L} \mathrm{blaze}=48^{\circ}$ -b $300 \mathrm{gr} / \mathrm{mm}$ J Y blaze $=17^{\circ}$

3) Spherical camera $\emptyset 200 \mathrm{~mm} \quad f=1000 \mathrm{~mm}$

Eig. 2 : Test spectrum

Detector : Photographic plate

Slit

: $21 \mu \mathrm{m}$

Grating : $600 \mathrm{gr} / \mathrm{mm} 4^{\text {th }}$ order

Dispersion : $2.9 \AA / \mathrm{mm}$ at $\mathrm{NaD}$

4) Flat Newton mirror

Resolution : $R=610^{4}$

5) Detector : CCD RCA or Thomson

6) Optical fiber

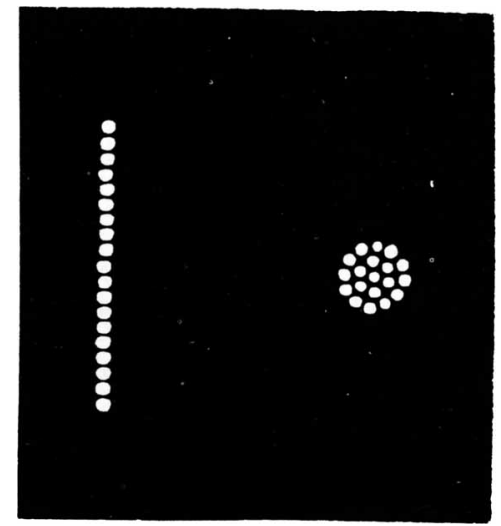

Eig. 3 : Fiber image slicer 19 glass fibers. $\varnothing 45-50 \mu \mathrm{m}$ $1=30 \mathrm{~mm}$

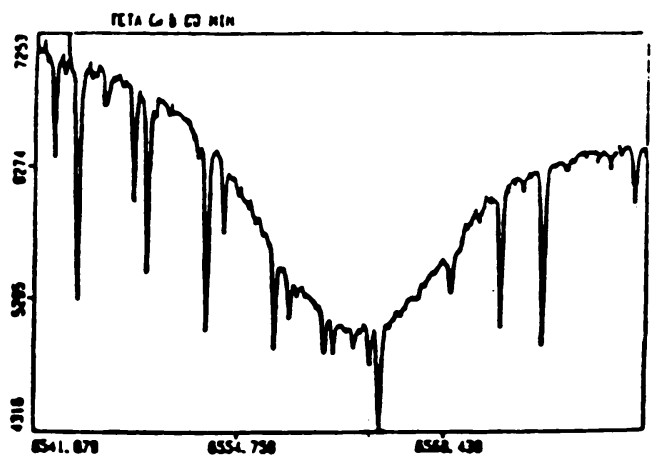

Fig. 4 : Resolution test on $\theta$ Cra $\mathrm{R}=3.510^{4}$ 


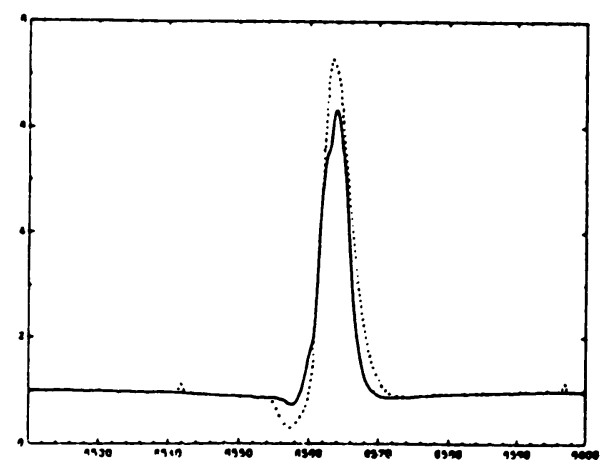

Fig. $5: H \alpha$ variability of $A B$ Aur $\left(m_{v}=7.2\right)$

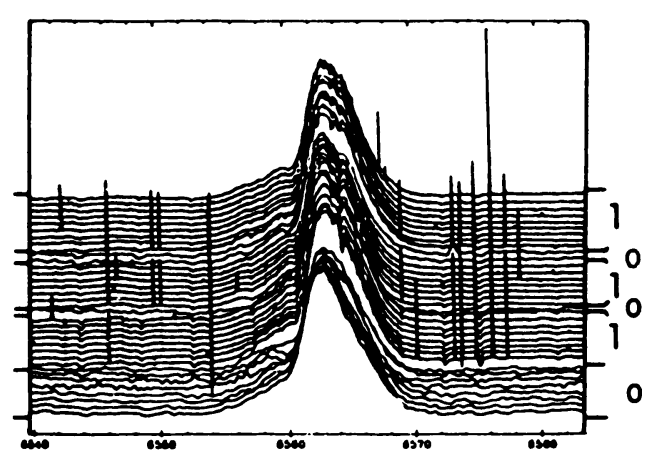

Fig. $6: \mathrm{H} \alpha$ bi-site observations of $\mathrm{BD}+46^{\circ} 3471$

However, when a CCD with $30 \mu \mathrm{m}$ pixels is used, it is possible to set the lines perpendicular to the dispersion by rotating the slit with an adequate angle, thus simplifying the reduction procedure. This is the actuel set-up used by observing visitors on ISIS at OHP. The dotted aspect of the spectral lines seen on Fig. 2, is due to the use of an fiber image slicer instead of a slit. The shape of the image slicer is presented on Fig. 3. The image slicer must be made of glass because silica fibers have a thick cladding leading to a poor packing efficiency.

The observations : The spectral capability of ISIS was checked on the sky in association with the $193 \mathrm{~cm}$ telescope at OHP. Fig. 4 shows a spectrum of $\theta \mathrm{Cra}$. The real spectral resolution is derived from this spectrum. The resolution is $3.510^{4}$ and the water vapor lines are clearly visible. This is a very important point, because when searching for spectral variability one has to be cautious about water vapor variation if insufficient resolution is employed. Fig. 5 shows the H $\alpha$ variation in AB Aur, a PMS Herbig Ae star with a resolution of $10^{4}$. ISIS was also used in a bi-site campaign on a rotational modulation search of the Herbig Ae star, BD+46 3471 . Fig. 6 shows the $\mathrm{H} \alpha$ variations for this star as seen from $\mathrm{OHP}(0)$ and $\operatorname{LICK}(1)$. A Fourier analysis of the data showed no periode in these variations.

Acknowledgments:We are pleased to thank G. Rousset and I. Raulet who where deeply involved in ISIS construction as well as $\mathrm{D}$. Gillet who is the astronomer in charge of ISIS at OHP. H. Hubert provided us with the $\theta$ Cra spectrum and M. Dreux and T. Fauconnier participated in the $\mathrm{BD}+46^{\circ} 3471$ observation at $\mathrm{OHP}$. 
Question by : I. Appenzeller

Could you give a number on the total efficiency of your spectrograph when working in the "image slicer mode", i.e. when observing stars with a matrix of many small diameter fibers?

Answer : With our present image slicer (19 fibers, $\varnothing 45-50 \mu \mathrm{m}, 30$ $\mathrm{mm}$ long) the gain over a slit is $35 \%$. We are making a new one, with only 7 fibers that is supposed to increase this gain.

Question by : Deshpande

Do the fibres introduce any polarization?

Answer: Our step index fibers do not introduce any polarization. To preserve polarization of propagated light, single mode fiber with special cladding should be employed.

\section{Question by: G. Marcy}

The nonuniform illumination of the input to the fiber at the telescope focal plane may cause nonuniform illumination along the slit. The nonuniform illumination is due to the stellar image not being as large as the fiber. Does this affect flat fielding ?

Answer : When using a long optical fiber, its output illumination is uniform and independent from the input position of the beam at the entrance face. This leads to an uniform illumination of the slicer whatever the position of the star at the entrance of the single fiber. 